\title{
Aceitabilidade dos medicamentos genéricos entre os alunos de um curso de farmácia do Norte de Minas Gerais
}

\author{
Acceptability of generic drugs among students of a pharmacy course in the North of Minas Gerais \\ Aceptabilidad de medicamentos genéricos entre estudiantes de un curso de farmacia en el norte de \\ Minas Gerais
}

Recebido: 07/11/2021 | Revisado: 13/11/2021 | Aceito: 17/12/2021 | Publicado: 01/01/2022

Joyce Soares Costa

ORCID: https://orcid.org/0000-0001-7649-8616 Faculdade Santo Agostinho, Brasil

E-mail: joyce_livesoares@hotmail.com

Matheus Borges de Oliveira

ORCID: https://orcid.org/0000-0001-6514-9824

Faculdade Santo Agostinho, Brasil

E-mail: matheusborges7806@gmail.com

Flavio Junior Barbosa Figueiredo

ORCID: https://orcid.org/0000-0003-1452-9573 Faculdade Santo Agostinho, Brasil E-mail: flavio@ fasa.edu.br

Thales de Almeida Pinheiro

ORCID: https://orcid.org/0000-0003-2228-6065 Faculdade Santo Agostinho, Brasil E-mail: thales@fasa.edu.br

Luis Paulo Ribeiro Ruas

ORCID: https://orcid.org/0000-0002-9356-503X Faculdade Santo Agostinho, Brasil E-mail: luis.paulo@fasa.edu.br

Thaísa de Almeida Pinheiro

ORCID: https://orcid.org/0000-0002-7599-3057

Faculdade Santo Agostinho, Brasil

E-mail: thaisafarma@yahoo.com.br

Talita Antunes Guimarães

ORCID: https://orcid.org/0000-0002-6627-9919

Faculdade Santo Agostinho, Brasil

E-mail: talitaa@fasa.edu.br

\section{Resumo}

A Lei 9.787/99 veio para marcar e difundir o medicamento genérico como idêntico ao de referência e de melhor acessibilidade aos usuários. Para tal, devem ser submetidos a testes de bioequivalência e biodisponibilidade para se tornarem intercambiáveis com aqueles de referência. Este trabalho tem como objetivo geral verificar a aceitabilidade dos medicamentos genéricos em alunos do curso de farmácia da faculdade Santo Agostinho - MG, bem como analisar possíveis motivos que interferem na adesão ou aceitação dos alunos no tratamento com os medicamentos genéricos. A metodologia utilizada tem caráter descritivo quantitativo, onde aplicou-se um questionário para coleta dos dados. A maioria dos alunos entrevistados afirmaram conhecer a Lei dos genéricos, sendo que aproximadamente $95 \%$ deles sabem a diferença entre os medicamentos de referência e genéricos e, $98,68 \%$ já fez uso de medicamentos genéricos. Em um panorama geral, a aceitabilidade dos medicamentos genéricos por parte dos alunos que se dispuseram a responder o questionário é alta; eles acreditam na eficácia dos medicamentos e aceitam a sugestão dos farmacêuticos quando indicam o medicamento genérico em substituição ao de referência prescrito. Conclui-se assim, que, ainda que relativamente recente, os medicamentos genéricos já são mais usados e aceitos pela sociedade, principalmente pelo seu preço mais acessível, facilitando adesão aos tratamentos que exigem o uso de medicamentos por períodos mais longos. A orientação e esclarecimentos oferecidos por parte dos farmacêuticos também é um fator importante nesta realidade, ressaltando a importância do profissional farmacêutico na promoção da saúde na sociedade.

Palavras-chave: Intercambialidade de medicamentos; Medicamentos Genéricos; Bioequivalência; Biodisponibilidade. 


\begin{abstract}
The law 9,787/99 came to mark and spread generic medicine as identical to the reference and with better accessibility to users. For that, they must submit bioequivalence and bioavailability tests to become interchangeable with those of reference. This research has as general objective to analyze the acceptability of generic drugs in pharmacy course students at Santo Agostinho College - MG, as well as to analyze possible reasons that interfere in the adherence or acceptance of students in the treatment with generic drugs. The methodology used has a quantitative descriptive character, where a questionnaire was applied for data collection. Most of the students interviewed claimed to know the Generics Law, and approximately $95 \%$ of them know the difference between reference and generic drugs, and $98.68 \%$ have already used generic drugs. Overall, the acceptability of generic drugs by students who were willing to answer the questionnaire is high; they believe in the effectiveness of medications and accept the suggestion of pharmacists when they indicate the generic drug instead of the prescribed reference. It is thus concluded that, although relatively recent, generic drugs are already more used and accepted by society, mainly for their more affordable price, facilitating adherence to treatments that require the use of drugs for longer periods. The guidance and clarification offered by pharmacists is also an important factor in this reality, highlighting the importance of the pharmacist in promoting health in society.
\end{abstract}

Keywords: Drugs Interchangeability; Generic Medicines; Bioequivalence; Bioavailability.

\title{
Resumen
}

La ley 9.787 / 99 vino a marcar y difundir el medicamento genérico como idéntico al de referencia y con mejor accesibilidad para los usuarios. Para ello, deben someterse a pruebas de bioequivalencia y biodisponibilidad para ser intercambiables con las de referencia. Este trabajo tiene como objetivo general analizar la aceptabilidad de medicamentos genéricos en estudiantes del curso de farmacia del Santo Agostinho College - MG, así como analizar posibles razones que interfieren en la adherencia o aceptación de los estudiantes en el tratamiento con medicamentos genéricos. La metodología utilizada tiene un carácter descriptivo cuantitativo, donde se aplicó un cuestionario para la recolección de datos. La mayoría de los estudiantes entrevistados afirmó conocer la Ley de Genéricos, y aproximadamente el $95 \%$ de ellos conoce la diferencia entre medicamentos de referencia y genéricos, y el 98,68\% ya ha usados medicamentos genéricos. En general, la aceptabilidad de los medicamentos genéricos por parte de los estudiantes que estaban dispuestos a responder el cuestionario es alta; creen en la eficacia de los medicamentos y aceptan la sugerencia de los farmacéuticos cuando indican el medicamento genérico en lugar de la referencia prescrita. Así, se concluye que, aunque relativamente reciente, los medicamentos genéricos ya son más utilizados y aceptados por la sociedad, principalmente por su precio más asequible, lo que facilita la adherencia a tratamientos que requieren el uso de medicamentos por períodos más prolongados. La orientación y aclaración que ofrecen los farmacéuticos también es un factor importante en esta realidad, destacando la importancia del farmacéutico en la promoción de la salud en la sociedad.

Palabras clave: Intercambiabilidad de fármacos; Medicamentos genéricos; Bioequivalencia; Biodisponibilidad.

\section{Introdução}

O Brasil começou a debater sobre os medicamentos genéricos na década de 70, porém na década de 90 que foi possível a regulamentação desses medicamentos através do Decreto 793, de 05/04/1993 revogado pelo Decreto 3.181, de 23/09/1999, que regulamentou a Lei $n^{\circ}$ 9.787, de 10/02/1999. Apenas com a publicação deste decreto, que foram criadas as condições para que ocorresse a fabricação e permissão da comercialização dos medicamentos genéricos no Brasil (ANVISA, 2020b).

Os primeiros 182 registros para produtos genéricos foram concedidos no ano 2000, quando também foram tomadas ações que implementaram sua produção, inclusive incentivando sua importação (ANVISA, 2020b). De uma análise de 284 lotes de medicamentos genéricos em 2016 e 2017, 85,9\% estavam de acordo as informações do fabricante e estavam cumprindo as exigências técnicas. Dados mostram que os medicamentos genéricos no Brasil passaram de 146, em 2014, para 336, em 2017, apresentando um aumento de 130,1\% em quatro anos, esse aumento vem mostrando que o mercado do genérico está em constante ascensão (ASCOM/ANVISA, 2018).

Até 05 de agosto de 2019, 5.723 medicamentos foram registrados, desse total, 2.398 tiveram seus registros anulados, restando um saldo de 3.325 medicamentos genéricos com registros válidos (ANVISA, 2020a). No período em questão, 120 empresas eram detentoras dos registros válidos, considerando que $85,6 \%$ dos medicamentos genéricos com registro válido são produzidos no Brasil. Do restante, cerca de, $8,3 \%$ são produzidos na Índia, 1,1\% na Alemanha, 0,7\% no Canadá, 0,3 na Itália e 
0,3\% nos Estados Unidos da América. Conforme informações do Conselho Federal de Farmácia (CFF), em 2016 o Brasil conquistou a sexta posição entre os maiores consumidores de medicamentos do mundo (CFF, 2016).

Os genéricos só podem ser vendidos e comercializados para a população depois de passarem por testes de equivalência farmacêutica e bioequivalência, comprovando sua eficácia, segurança e qualidade. Somente após passarem por estes testes que eles podem ser intercambiáveis. Eles devem conter o mesmo princípio ativo, dose, forma farmacêutica, a mesma via de administração e deve ser indicado para o mesmo tratamento quando comparado com o medicamento de referência. É isento de nome comercial e seu valor deve ser 35\% mais barato quando comparado ao de referência. Suas embalagens precisam constar uma tarja amarela com a letra "G" e a indicação "Medicamento Genérico". Estes medicamentos podem assumir o lugar dos medicamentos de referência, sendo, portanto, considerados intercambiáveis (Lista, 2018).

O desconhecimento a respeito do que realmente são os medicamentos genéricos, pode levar muitas pessoas a rejeitar o uso desses medicamentos, devido à baixa compreensão do conceito que os envolve. É valido desmembrar aqui alguns conceitos que podem levar a um melhor entendimento sobre as classes de medicamentos de uma maneira geral. Medicamentos de referência são aqueles comercializados sob um nome comercial ou de marca, que apresentam patentes que os resguardam quanto à exclusividade de produção daquele produto por tempo determinando, com objetivo de se reaver todo investimento feito no desenvolvimento, estudo e distribuição daquele medicamento; já os medicamentos genéricos, não possuem patente e sua comercialização se dá através do nome do princípio ativo do medicamento em questão, porém, segue os mesmo padrões de qualidade, produção e bioequivalência de um produto de referência (Manhães \& Hasenclever, 2018).

O quesito que diz respeito à diferença de valor entre os medicamentos de marca e os genéricos, está exatamente no fato de que, para os medicamentos genéricos não houve nenhum investimento inicial de inovação e/ou criação do medicamento, visto que estes foram realizados pela marca que detinha sua patente. Há ainda os medicamentos similares, são produtos que, em tese apresentam mesma função e efeitos, no entanto, diferente da classe anteriormente citada, estes não apresentam exigência de testes de bioequivalência e biodisponibilidade em relação ao medicamento de referência (Manhães \& Hasenclever, 2018).

A intercambialidade é garantida por meio de testes de equivalência terapêutica, que incluem comparação in vitro, através de estudos de equivalência farmacêutica, e in vivo, com os testes de bioequivalência apresentados à Agência Nacional de Vigilância Sanitária (ANVISA, 2018a). A diminuição do preço dos medicamentos genéricos é possível, pois os fabricantes não investem no marketing, além de não precisarem fazer ensaios clínicos, pois os mesmos já foram realizados pela indústria patenteadora (Blatt et al., 2012).

\subsection{Intercambialidade e a importância do profissional de farmácia}

Os medicamentos genéricos podem ser fabricados somente após o final do prazo da proteção da patente do medicamento original. São cópias que seguem as fórmulas que já existem e, pelo fato de os fabricantes não precisarem fazer altos investimentos em pesquisa e desenvolvimento, chegam aos consumidores com valores bem mais baixos. Assim população tem maior possibilidade de adquirir medicamentos seguros, eficazes e de qualidade. Isto porque os medicamentos genéricos somente podem ser colocados no mercado após serem aprovados em testes de equivalência farmacêutica e bioequivalência, segundo as normas da ANVISA (PRÓ-GENÉRICOS, 2018).

A construção deste trabalho consiste na análise da jornada da promulgação da lei que regulamenta os genéricos no Brasil, bem como a importância da intercambialidade entre os princípios ativos que precedem as evidências que apontam a boa aceitação da política brasileira de incentivo à produção final dos medicamentos genéricos. Nos últimos 21 anos o governo regulamentou os medicamentos genéricos aqui no Brasil por meio da Lei 9.787, e essa regulamentação tem por conquista um marco nas políticas públicas relacionadas à saúde dos brasileiros, como garantia de acesso aos remédios por todos os usuários 
do SUS (Sistema Único de Saúde). Hoje, muito dos consumidores que optam pelo genérico tem consciência em sua eficácia e segurança, isso é considerado um avanço no acesso de medicamentos de qualidade e de baixo custo (ANVISA, 2019). A importância da aceitação do farmacêutico nessa trajetória deve ser relevante, de maneira indissociável, ele é quem participa ativamente em todo processo que diz respeito aos testes de bioequivalência e biodisponibilidade.

A intercambialidade consiste em optar entre dois medicamentos com a mesma finalidade terapêutica, capaz de garantir igualdade em eficácia e segurança no tratamento, atingindo os resultados terapêuticos desejados (Torres \& Chinchila, 2012).

Para Guttier et al., (2016), equiparam a percepção, o conhecimento e uso dos medicamentos genéricos entre os anos 2002 e 2012 no sul do Brasil e declararam que a convicção sobre a qualidade dos medicamentos genéricos é um motivo que pode induzir na sua utilização, pois com um maior conhecimento e uma experiência prévia com o medicamento genérico percebeu que isto é um fator de extrema importância na compreensão da qualidade que tem esses medicamentos. À medida que os consumidores adquirem uma maior bagagem com os medicamentos genéricos, estes passam a comprar e admitir sua qualidade, sendo mais bem aceitos e utilizados pela população.

Apesar da importância ainda há certo nível de resistência e insegurança entre a população e principalmente entre os profissionais de saúde, uma vez que o maior fator de resistência à utilização do medicamento genérico é devido ao pouco conhecimento dos consumidores, o baixo estímulo dos prescritores e a ausência de orientação sobre o uso desses medicamentos (Moraes, 2016).

A seleção de um produto, inclusive de medicamentos genéricos, é induzida por diversos fatores, podendo ser citado principalmente os prescritores, familiares e o farmacêutico na escolha pelo medicamento a ser consumido. O profissional prescritor é o responsável pelo diagnóstico e tratamento e possui uma relação mais próxima com o paciente, sendo, portanto, o profissional que o paciente tem maior confiança. O farmacêutico, sendo o profissional que pode fazer a intercambialidade na prescrição, possui um importante papel no comércio destes medicamentos, atuando de forma eficaz como um mediador na aquisição destes medicamentos (Novaretti et al., 2014).

Portanto o objetivo deste estudo é verificar o grau de aceitação dos estudantes do curso de Farmácia da Faculdade Santo Agostinho, na cidade de Montes Claros e mostrar se os estudantes estariam dispostos a realizar a troca de medicamentos de referência por medicamentos genéricos, identificando os possíveis motivos que interferem na adesão ou aceitação, a confiabilidade dos participantes na realização da intercambialidade dos medicamentos e o conhecimentos destes sobre a evolução dos medicamentos genéricos, através da aplicação de um questionário para obter estes dados

\section{Metodologia}

Este estudo possui caráter descritivo, de corte transversal e análise quantitativa de campo, que objetivou verificar o grau de aceitação dos estudantes do curso de Farmácia da Faculdade Santo Agostinho, na cidade de Montes Claros (Cruz et al., 2021). Realizou-se um estudo, onde 152 alunos do curso de farmácia da Faculdade Santo Agostinho (situada em Montes Claros - MG) foram convidados a responder o questionário, após explicação sobre o tema nas turmas do referido curso.

Foram incluídos na pesquisa todos os alunos maiores de 18 anos que, de forma voluntária, assinaram o Termo de Consentimento Livre e Esclarecido e que estão devidamente matriculados na instituição de ensino. A coleta de dados com a aplicação do questionário aconteceu entre os meses de julho e setembro de 2021, após aprovação do projeto pelo Comitê de Ética em Pesquisa (CEP), sob parecer nº. 4.687.344, submetido via Plataforma Brasil. O mesmo contém 11 questões objetivas, com o intuito de analisar a aceitação dos genéricos, bem como o nível de conhecimento em relação à intercambialidade de medicamentos genéricos e de referência. A metodologia adotada foi baseada no mesmo método utilizado por, no desenvolvimento de seus estudos à respeito dos fatores associados à aceitação dos medicamentos genéricos pela população. 
Para minimizar qualquer constrangimento ou desconforto ao participante, o questionário será mantido de forma anônima, sendo que cada aluno respondeu o questionário individual e pessoalmente e, somente o pesquisador tem acesso às informações fornecidas pelo aluno, sendo assim garantido o sigilo de sua participação e informações na pesquisa, podendo o mesmo decidir por não continuar a responder o questionário a qualquer momento durante a pesquisa. A análise dos dados foi realizada no programa de Microsoft Excel, onde foram tabulados e os resultados apresentados em gráficos e tabelas obtidas com a aplicação do questionário

\section{Resultados}

A amostra constituiu-se de 152 indivíduos que assinaram o TCLE e se dispuseram a responder o questionário aplicado, sendo a maior parte mulheres $(71,05 \%, n=108)$. Os participantes estão distribuídos entre $1^{\circ}(n=7), 2^{\circ}(n=34), 4^{\circ}$ $(\mathrm{n}=35), 5^{\circ}(\mathrm{n}=1), 6^{\circ}(\mathrm{n}=45), 8^{\circ}(\mathrm{n}=1), 9^{\circ}(\mathrm{n}=19)$ e $10^{\circ}(\mathrm{n}=10)$ períodos do curso de Farmácia na Faculdade Santo Agostinho, em Montes Claros - MG.

Com base nos dados obtidos com a aplicação do questionário, dispostos no Quadro 1, 59,21\% dos alunos tem conhecimento sobre a Lei dos Genéricos, que foi implementada em 1999, com objetivo de ampliar o acesso da população a tratamentos seguros, eficazes e mais baratos (BRASIL, 1999). Tem-se ainda que, 94,74\% destes sabem a diferença entre os medicamentos genéricos e as demais categorias de medicamentos (referência, similar, manipulado, alopático, homeopático, fitoterápico).

Quadro 1 - Distribuição das respostas obtidas através dos questionários aplicados para avaliar a aceitabilidade dos medicamentos genéricos em alunos do curso de farmácia.

\begin{tabular}{|lll|}
\hline Questionário & Respostas & n (\%) \\
\hline Você conhece a lei 9.787, conhecida como "lei dos genéricos"? & Sim & $90(59,21 \%)$ \\
\hline $\begin{array}{l}\text { Você sabe a diferença entre os genéricos e as demais } \\
\text { categorias de medicamentos? }\end{array}$ & Sim & $62(40,79 \%)$ \\
\hline Você utiliza ou já utilizou algum medicamento genérico? & Sim & $8(5,26 \%)$ \\
\hline $\begin{array}{l}\text { Se você já utilizou medicamentos genéricos, ele cumpriu com } \\
\text { o efeito desejado? }\end{array}$ & Sim & $150(98,68 \%)$ \\
\hline Você confia na eficácia dos medicamentos genéricos, quando & Sim & $144(1,32 \%)$ \\
comparados com medicamentos de marca? & Não & $8(5,26 \%)$ \\
\hline Você considera que o medicamento genérico é mais barato que & Sim & $139(8,55 \%)$ \\
o medicamento de referência? & Não & $6(3,95 \%)$ \\
\hline Você encontra o medicamento genérico que procura com & Sim & $142(93,42 \%)$ \\
facilidade na farmácia? & Não & $10(6,58 \%)$ \\
\hline Seu médico costuma prescrever medicamentos genéricos? & Sim & $71(46,71 \%)$ \\
\hline Você aceita que o farmacêutico faça a troca de um & Sim & $81(53,29 \%)$ \\
medicamento de referência pelo genérico? & Não & $136(10,53 \%)$ \\
\hline
\end{tabular}

Fonte: Autores (2021). 
Ao serem questionados quanto ao uso do medicamento genérico, apenas 1,32\% afirmou não utilizar ou não ter feito uso desses medicamentos, sendo que, ainda assim, 8,55\% informou não confiar na eficácia dos medicamentos genéricos em comparação com os medicamentos de referência. 96,05\% dos participantes relataram que consideraram o medicamento genérico é mais barato que o medicamento de referência e $93,42 \%$ tem facilidade em encontrar o medicamento genérico que precisam com facilidade nas farmácias.

Quando questionados sobre a prescrição de medicamentos genéricos, 46,71\% afirmaram que os seus médicos prescrevem medicamentos da classe e 53,29\% não recebem esse tipo de prescrição. $E$, no que diz respeito à troca do medicamento de referência pelo medicamento genérico por parte do farmacêutico responsável pelo atendimento, apenas $10,53 \%$ não aceitam que esta troca seja realizada.

\section{Discussão}

Uma parcela considerável dos estudantes participantes afirmou desconhecer ainda da lei mencionada. No entanto, pode-se levar em consideração que, aproximadamente, metade destes estão cursando, ainda, a primeira metade do ensino superior em Farmácia e, apesar de não conhecerem a lei, a maioria afirmou saber a diferença entre os medicamentos genéricos e os demais.

A Lei $9.787 / 99$ foi implementada a fim de regulamentar a utilização de medicamentos genéricos por parte da população, com melhores preços de compra, bioequivalência e intercambialidade com os medicamentos de referência. Esta lei proporcionou aos pacientes/usuários dos medicamentos uma maior adesão aos tratamentos devido ao custo mais baixo, sem perda de eficácia esperada (Remedio \& Prado, 2019).

Blatt et al. (2012), tiveram como objetivo pesquisar o nível de conhecimento e grau de utilização de genéricos em residentes do município de Tubarão, em Santa Catarina, em que quase 90\% dos entrevistados afirmaram já terem feito uso de medicamentos genéricos e se mostraram satisfeitos com os resultados. Apesar da concordância dos estudos de Blatt $e t$ al. com a presente pesquisa apresentada, é possível observar ainda usuários que não confiam na eficácia dos medicamentos genéricos em comparação com medicamentos de marca. Sebben e Fernandes (2019), compilam em seu estudo diferentes dados sobre o conhecimento e aceitação dos medicamentos genéricos por usuários, em uma revisão integrativa, onde foi possível concluir que houve um crescente conhecimento, aceitação e uso dos genéricos ao longo dos anos, devido a inúmeras ações de propaganda para conhecimento.

Ainda que o percentual de alunos que não confiam na eficácia dos medicamentos genéricos neste estudo $(8,55 \%)$ ser baixo, este cenário é ainda muito real na população em geral. Estudos apontam que os principais motivos para as pessoas não fazerem uso destes medicamentos são: a crença de que por ser mais barato, é também menos eficaz ou ter menor qualidade, apesar de apresentar mesma composição (dose, princípio ativo e apresentação) são mais "fracos" que o de referência, sendo necessário uma dose maior para se ter um mesmo efeito e apresentarem mais efeitos colaterais. O desconhecimento quanto aos testes à que os medicamentos genéricos são submetidos para assegurar eficácia, qualidade e segurança e aprovação da ANVISA, pode levar a opiniões equivocadas, subestimando a qualidade do produto, reduzindo a adesão dos usuários (Sebben \& Fernandes, 2019). Jarczewski, Ferreira \& Dal Piva (2019), apontam ainda que a confiabilidade sobre o medicamento genérico sofre interferência da baixa qualidade de alguns medicamentos similares, vinda da opinião de consumidores de alguns anos atrás, apesar da modificação das leis brasileiras a respeito da exigência para produção destes medicamentos.

Um dos principais fatores para regulamentação e produção de medicamentos genéricos é o preço de venda mais baixo, mantendo a qualidade e segurança do medicamento. Pouco mais de $96 \%$ dos entrevistados neste estudo afirmaram que os medicamentos genéricos são mais baratos que o de referência. Este é o fator relatado como maior vantagem a ser considerada no momento da aquisição dos medicamentos, visto que, muitas vezes o medicamento prescrito pelos médicos é o de referência 
e, caso os preços fossem iguais, os pacientes teriam preferência por adquirir o medicamento de referência, conforme prescrito (Jarczewski, Ferreira \& Dal Piva, 2019).

Devido a disseminação da lei e campanhas acerca da utilização de medicamentos genéricos, não há muita dificuldade por parte dos usuários em encontrar medicamentos genéricos nas farmácias. Além disso, alguns outros fatores são apontados como facilitadores da difusão destes medicamentos no mercado brasileira: programas do governo (Farmácia Popular), aceitação e confiança dos consumidores quanto à qualidade dos medicamentos, prescrição médica pelo princípio ativo e a aumento do número de patentes expiradas. Todos estes fatores citados, causam um aumento na produção, na procura e, consequentemente, na disponibilidade destes produtos nos balcões e farmácias e unidades de saúde (Souza, 2020).

No que diz respeito à prescrição dos medicamentos pelo nome do seu princípio ativo, 53,29\% afirmaram que os médicos que os atendem ainda não adotaram esta prática. Souza (2020), traz ainda à discussão, o fato de que a prescrição médica tem impacto importante no elemento de relação entre as empresas farmacêuticas, médicos, consumidores e governos, visto que sua influência sobre a demanda é mais intensa sobre os produtos de referência. A escolha do medicamento é feita pelo médico prescritor, não pelo consumidor, que por sua vez não é responsável financeiramente pela aquisição do produto. $\mathrm{O}$ grande investimento das industrias farmacêuticas em marketing para com seus clientes (médicos e farmácias) garante a eles, também, uma certa lealdade por parte dos seus clientes, garantindo assim maiores lucros, mesmo em situações que expiração das patentes de exclusividade (Souza, 2020).

Apesar de todos os fatores contrários ao uso de medicamentos genéricos que foram apresentados durante o estudo, aproximadamente $90 \%$ dos participantes aceitam que o farmacêutico faça a troca do medicamento de referência pelo medicamento genérico. Isso indica uma maior e crescente aceitação por parte da população, dos medicamentos genéricos, foco deste estudo, levando também em consideração o grau de informação disponibilizado para estes usuários. Nos estudos desenvolvidos por Silva, Bonfim \& Oliveira (2020), a grande maioria dos usuários aceitam os genéricos e levam em consideração a indicação farmacêutica sobres estes, e no que se refere à intercambialidade entre o de referência e o genérico, por interferência do profissional farmacêutico, $95 \%$ não se opõe a esta troca. Vê-se que o profissional farmacêutico tem papel importante na orientação sobre o uso de medicamentos e dúvidas no que cerca a oferta dos medicamentos genéricos à população. De forma responsável, o farmacêutico é habilitado para fazer a indicação e intercambialidade dos medicamentos, de modo tranquilo e sem oposições, visto que, ainda hoje, muitas vezes o farmacêutico é o primeiro profissional procurado pela população para tratamento de "males menores" e auxílio no entendimento das orientações médicas (Silva, Bonfim \& Oliveira, 2020).

\section{Conclusão}

Pode-se concluir com este estudo, que ainda que a Lei dos genéricos seja relativamente recente, eles já são melhores aceitos e apresentam um uso maior pela população. Uma grande parte dos alunos entrevistados faz ou já fez uso de medicamentos genéricos e obtiveram resultados satisfatórios. Ainda que, por parte da classe médica, com base nos dados obtidos, a prática da prescrição de genéricos não seja tão frequente, grande parte dos indivíduos aceitam que o farmacêutico faça a substituição do medicamento prescrito pelo genérico, pratica que gera até uma certa facilidade para o usuário, pelo fato desses medicamentos serem mais facilmente encontrados nas farmácias e drogarias. Outro fator que acredita-se levar as pessoas a aderirem ao uso destes medicamentos, é a orientação e esclarecimentos recebidos nas unidades de saúde por parte dos farmacêuticos, ressaltando a importância do profissional farmacêutico na promoção da saúde na sociedade.

A atenção farmacêutica é um assunto importante ainda pouco difundido e de extrema valia para os usuários dos sistemas de saúde, uma vez que a sociedade sente confiança nos profissionais para orientações a respeito do uso dos medicamentos. Esta atividade permite uma relação direta do farmacêutico com os usuários dos medicamentos, a fim de uma se 
atingir uma farmacoterapia racional, visando melhor adesão e resultados nos tratamentos, obtendo assim, melhoria na qualidade de vida.

\section{Referências}

ANVISA (2019). ANVISA destaca Dia Nacional do Medicamento Genérico. https://www.gov.br/anvisa/pt-br/assuntos/noticias-anvisa/2019/anvisa-destacadia-nacional-do-medicamento-generico.

ANVISA (2020a). Medicamentos Genéricos. https://www.gov.br/anvisa/pt-br/assuntos/medicamentos/genericos/genericos.

ANVISA (2020b). Medicamentos genéricos: estatísticas e listas. https://www.gov.br/anvisa/pt-br/assuntos/medicamentos/genericos/estatisticas.

ASCOM/ANVISA (2018). Registro de Medicamentos Genéricos faz 18 anos. https://url.gratis/pIRMlhttps://url.gratis/pIRM14.

Blatt, C. R. et al (2012). Conhecimento popular e utilização dos medicamentos genéricos na população do município de Tubarão, SC. Revista Ciência \& Saúde Coletiva, 17 (1).

BRASIL (1999). Lei $n^{\circ}$ 9787, de 10 de fevereiro de 1999. Disponível em: http://www.planalto.gov.br/ccivil_03/leis/L9787.htm.

CFF (2016). Farmácias de elite. http://www.cff.org.br/noticia. php?id=3879.

Cruz, A. F. P. et al. (2021). Fatores associados à aceitação dos medicamentos genéricos pela população. Research, Society e Development. 10 (10).

Guttier, M. C. et al., (2016). Percepção, conhecimento e uso de medicamentos genéricos no sul do Brasil: o que mudou entre 2002 e 2012?. Cad. Saúde Pública, Rio de Janeiro, 32(7).

Jarczewski, F.; Ferreira, S. C. \& Dal Piva, R. (2019) Avaliação sobre o conhecimento a respeito de medicamentos genéricos em uma amostragem de usuários de medicamentos no município de São Miguel do Iguaçu - Paraná. Revista UNINGÁ, 56 (3), 106-121.

LISTA de Medicamentos Genéricos (2018). O que são genéricos? http://medicamentogenerico.com.br.

Magalhães, B. S. \& Rocha, M. S. (2016). A presença do medicamento similar na intercambialidade. Revista Oswaldo Cruz. 3 (11), 2-4

Manhães, E. \& Hasenclever, L. (2018). A territorialidade da política pública dos medicamentos genéricos no Brasil - 2000/2017. Revista de Políticas Públicas, 22 (2), 987-1008.

Moraes, S. L. C. S. (2016). Avaliação do perfil e aceitação de medicamento genérico de clientes de uma drogaria na cidade de Quirinópolis - GO. http://www.unirv.edu.br/conteudos/fckfiles/files/AVALIACAO\%20DO\%20PERFIL\%20 E\%20ACEITACAO\%20DO\%20MEDICAMENTO.pdf.

Novaretti, M. C. Z.; Quitério, L. M. \& Piscopo, M. R. (2014). Desafios da gestão de medicamentos genéricos no Brasil: da produção ao mercado consumidor. Rio de Janeiro/RJ 13 a 17 de setembro de 2014.

Pró-genéricos (2018). Medicamento genérico: uma importante conquista para a saúde pública no Brasil. http://www.progenericos.org.br/pdf/Medicamento_Generico_15anos.pdf.

Remedio, J. A. \& Prado, C. C. V. (2019). Lei de propriedade industrial: parágrafo único do artigo 40 da Lei 9,279/96 e acesso a medicamentos genéricos. Revista Direito em Debate. 38 (52), 115-124.

Sebbem, S. N. B. \& Fernandes, L. C. (2019). Conhecimento e aceitação dos medicamentos genéricos por usuários: uma revisão integrativa. Revista Destaques Acadêmicos, 11 (3), 211-229.

Silva, A. E. P.; Bonfim, A. J. \& Oliveira, F. C. B. (2020). Utilização de medicamentos genéricos em um estabelecimento farmacêutico do município de Teresina (PI). E-Revista, 18. http://periodicos.estacio.br/index.php/e-revistafacitec.

Souza, C. M. A.(2020) A regulação do preço dos medicamentos genéricos no Brasil. Universidade Federal do Rio de Janeiro (dissertação - mestrado). 136 f.

Torres, N. P. B. \& Chinchilla, I. N. (2012) Medicamentos Similares e Genéricos: Biodisponibilidade Relativa e Contexto Regulatório do Cenário Brasileiro Atual. RRS - FESGO 2 (2). 